\title{
Global Health: Global Infectious Disease Control Systems
}

\author{
Shinu Kuriakose* \\ Department of Physician Assistant Studies, USA
}

*Corresponding author: Shinu Kuriakose, Department of Physician Assistant Studies, USA.

Received Date: September 27, 2019

Published Date: October 30, 2019

\section{Introduction}

The fundamental issue concerning global healthcare policy is the recognition of the role various institutions, including nongovernmental agencies and countries, play to mitigate the spread of diseases and other pathological factors to prevent global morbidity and mortality. It is inherent that the role of not only screening, reporting, diagnosing and treating illness, with a potential to be a pandemic, be have clear command and control protocols but also timeliness and efficiency of this process is crucial. Poverty, maternal and child illnesses, disease prevention, vaccinations, and the paucity of food and water do play a crucial role for entities while framing foreign policy and regulations. The concept of rapid response to diseases, such as Ebola recently, can help reduce the number of casualties in a given population and furthermore help halt the spread of illness. It is the responsibility of the both the public and private sector to implement procedures in a manner with the understanding that a failure on their part could lead to disastrous consequences; a fact which lends itself to the recognition that the world is increasingly inter-connected and an ill person can just as easily spread a disease in New York, due to the immediacy and availability of air transport as $\mathrm{h} / \mathrm{she}$ can spread it in their neighboring village in Asia.

The Oslo Declaration in 2010, signed by the foreign ministers of seven countries, has in its core the concept of how any proposed policy will affect healthcare in the region and important consideration must also be given to economic consequence of healthcare reform, collaboration between parties and the implementation of healthcare protection to provide sound medical protection on a global scale. Furthermore, The Disease Control Priorities Project (DCP2), in 2006, has attempted to formulate questions and responses a country must ask itself to assess its preparedness for an health outbreak; a process which takes into consideration the universal definition of a disease, the effectiveness and response to this malady, the priorities which need to be considered in rank order, the interventions which need to be implemented to achieve said objectives, the importance of cost-effective processes taking into consideration equitable health care with a focus on reducing disparities, preventative strategies focusing on food and water safety, vaccination and immunization protocols and effective sanitation and educational programs [1].

\section{Discussion}

\section{World health organization}

The World Health Organization is a part of the United Nations, founded in 1948, with a specific focus and emphasis on public health internationally. This organization is currently focused on the eradicating of communicable diseases such as Ebola, HIV and TB and has been successful in the elimination of smallpox [2]. WHO characterizes diseases into categories such as communicable (viral or bacterial), non-communicable (obesity and mental health and injuries (accidental or natural disasters) [3]. WHO's priorities include supporting its member states in preparing their singular capacities to deal with epidemics and ensuring that enough warning systems are in place to screen for pathologies including laboratory facilities, implementing training programs for epidemic readiness, coordination among its members to deal with any pandemics and seasonal influenza, ensuring uniform protocols are in place to standardize both screening and treatment techniques and minting a ready stance to deal with any major outbreaks and providing support in the healthcare realm to its constituents [4].

In the recent Ebola outbreak, WHO played a pivotal role in the initial screening and understanding of this disease by sending its epidemiologists and health promotion officers, in early June 2014, to countries in West Africa where reports were emerging of a viral 
disease with lethal consequences. Its medical team had to track this illness to its source and look at the cumulative data from laboratories, patients, providers and hospitals to gain a better understanding of their healthcare adversary. Furthermore, WHO took the lead in preventing transmission of this disease by identifying folks with this illness, treating patients with Ebola, conducting safe burials and being the lead in prevention of spread of Ebola. Additionally, it attempted to halt the spread of Ebola in unaffected countries, deployed rapid response teams for new outbreaks, helped prepare unaffected countries in case of an outbreak, helped with infrastructure to isolate patients with this illness, helped develop rapid screening and treatment modalities and put resources in for research purposes in the realms of vaccinations for this disease [5].

\section{Centers for disease control and prevention}

The Centers for Disease Control and Prevention (CDC), founded in 1946, is the leading federal agency of the United States government, under the auspices of the Department of Health and Human Services, which deals with public health concerns. The CDC's primary agenda is more nationalistic in nature and focused on the prevention of disease and disability of United States citizens in areas of public health such as infectious disease, occupational safety issues, environmental health, and food borne pathogens and in chronic illnesses such as diabetes and hypertension [6]. The CDC wears multiple hats when dealing with an outbreak like Ebola which has primarily been affecting West Africa by advising healthcare workers who treat Ebola patients in these countries, by assisting hospital not only in the United States but also abroad including West Africa on standardized approaches to treat Ebola victims, on advising travelers and students who may travel to these countries for medical missions or tourism purposes, on assisting providers and lay folks on the signs and symptoms of Ebola, including time frames where these symptoms may occur, so that timely screening, diagnosing and treating can begin. In addition, the CDC has been in the forefront of helping US hospital in preparing for Ebola by having specialized units built in case of an outbreak and training clinicians on best practices to handle Ebola patients including the usage of personal protective gear. Furthermore, the CDC has been advising hospital on how to sterilize equipment and rooms formerly used for Ebola patients and advising foreign governments on ways the transmission of Ebola can be halted including basic things such as hand washing techniques and appropriate breast-feeding to prevent further dissemination to babies by infected mothers [7].

\section{Governmental Response}

The countries most affected by Ebola in West Africa, including Guinea, Liberia and Sierra Leone, have with the assistance of WHO, CDC and non-governmental organizations, have focused on the social underpinnings in their lands which has led this virus to flourish including lack of a robust health system, poor governmental organization and oversight, poverty and political conflict [8]. There is increased emphasis on enhanced screening of this disease, allowing international medical experts and agencies to come into their countries and evaluate the healthcare realities on the ground, assist agencies by providing the minimal resources they do have to help combat this epidemic. Furthermore, there is increased emphasis that better health system with more diligent screening and elimination of social stigma could encourage folks to treat care and enhance recovery efforts when dealing with this virus. Education also is key, as these nations have all suffered with the portrayal of HIV/AIDS destroying the lives of their citizens and the negative affect this can have on trade and tourism. The United States, with the assistance of the United Nations and other developed countries, has spent resources both medical, financial and social to help alleviate some of the difficulties this part of the world is facing with varying success; an attempt worth doing to help lay the ground for a more resourceful healthcare model for the next potential epidemic.

\section{Summary}

The challenges in front of the $\mathrm{CDC}$, WHO, governmental organizations can seem at times insurmountable but through vigilance and dealing in a timely manner with issues such as Ebola can help mitigate this situation in the long term. In a bid to prevent a pandemic from occurring, these organizations have taken upon themselves, with assistance from other non-governmental sources to tackle this problem in an effective manner. Although, thousands of lives were lost in this epidemic, timely intervention did prevent it from evolving into a worldwide pandemic. It is imperative that coordination, as seen in West Africa ravaged by Ebola, can play a crucial part in not only educating citizens to seek care if stricken by disease but also to encourage change on a fundamental level to the social structure of these countries to remove the stigma associated with disease.

\section{Recommendations}

It is my firm belief that as the world gets increasingly interconnected, there will be an enhanced likelihood that diseases will spread faster across the globe in not tackled at the onset. It might be prudent to develop a rapid response disease team which can go anywhere in the world in 48 hours to deal with any potential calamity. Yes, this will require substantial resources but our options remain limited in this endeavor. Another option could be having a team like this based on every continent so the response time can be quicker, however this might lead to developed countries having teams with more resources and could lead to health disparities. Overall, it is in the best interests of all to ensure that timely universal healthcare be available to all the citizens of this world lest we falter to a pandemic with no regard to social standing.

\section{Acknowledgment}

None.

\section{Conflict of interest}

No conflict of interest.

\section{References}

1. Disease Control Priorities Project (2015) About DCP2: Economic Evaluation for Health 
2. World Health Organization (2015) Ebola Diaries: Creating ways to understand an outbreak.

3. Jacobsen K (2014) Introduction to Global Health. (2 ${ }^{\text {nd }}$ edn), Sudbury, Jones and Bartlett Publishing Ltd, Mass, USA.

4. World Health Organization (2015) Ebola Diaries: Creating ways to understand an outbreak.

5. World Health Organization (2015) Ebola: Current Situation.
6. Centers for Disease Control and Prevention (2015) Saving Lives, Protecting People.

7. Centers for Disease Control and Prevention (2015) Ebola (Ebola Virus Disease).

8. United Nations Developmental Program (2015) Ebola Response Strategy for Guinea, Liberia and Sierra Leone. 